Check for updates

Cite this: RSC Adv., 2017, 7, 39011

\title{
Microbial consortium OEM1 cultivation for higher lignocellulose degradation and chlorophenol removal†
}

\author{
Jiajin Liang, (D) Yunqin Lin, ${ }^{*}$ Tian Li and Fanyong Mo \\ Herein, microbial consortium OEM1 that could simultaneously decompose lignocellulose and \\ chlorophenols was screened from spent mushroom substrates. Its degradation potential further needs to \\ be confirmed by optimizing the culture conditions (such as organic loads, initial pH values, and \\ cultivating ways). The results illustrated that $2.0 \%$ rice straw load provided highest organic degradation \\ rate with OEM1. After 9 days of cultivation, O-chlorophenol (MCP), 2,4-dichlorophenol (2,4-DCP), and \\ 2,4,6-trichlorophenol (2,4,6-TCP) were almost completely degraded. After 15 days, cellulose, \\ hemicellulose, and lignin were degraded by $97.4 \%, 76.4 \%$, and $69.8 \%$, respectively. The mass loss of the \\ rice straw could reach $66 \%$ when the initial $\mathrm{pH}$ value of the culture medium varied from 5.0 to 8.0. \\ Moreover, higher degradation efficiencies in chlorophenols were obtained under neutral or alkaline \\ condition. In addition, the degradation efficiency for lignocellulose and chlorophenol could reach $57.1 \%$ \\ and $58.6 \%$, respectively, with static cultivation.
}

Received 26th April 2017

Accepted 26th July 2017

DOI: $10.1039 / \mathrm{c} 7 \mathrm{ra0} 4703 \mathrm{~g}$

rsc.li/rsc-advances

chlorophenol solutions in the application process. Thus, these

\section{Introduction}

With the increased paper production, the broader application of activated sludge wastewater treatment process in pulp and paper mills has amplified the sludge management problem. ${ }^{1}$ It is estimated that nearly 10000 paper mills all over the world produce around 18 million tons of dry pulp and paper sludge (PPS) annually. ${ }^{2}$ As a typical biomass solid waste, PPS contains protein (22-52\%), lignin (20-58\%), carbohydrate (0-23\%), lipid $(2-10 \%)$, and cellulose $(2-8 \%)$. These polymers are difficult to be degraded and utilized via biological treatment. In addition, chlorine dioxide as an elemental chlorine free (ECF) bleaching agent is widely used in pulp and paper mills. ${ }^{3}$ Adsorbable organic halides (AOX) appear as a result of the reaction between residual lignin from wood fibers and chlorine/chlorine compounds originated during the bleaching process. ${ }^{4}$ Many of these compounds are very difficult to be degraded and have long half-life periods. Some of them tend to bio-accumulate, whereas some are proven mutagens and carcinogens. ${ }^{4}$ AOX finally migrate to the PPS in the wastewater treatment process. Hence, these compounds become a major bottleneck in the treatment and utilization of PPS. Moreover, chlorophenols as important chemicals were widely used as wood preservatives and herbicides. The lignocellulosic biomass was dipped in

College of Natural Resources and Environment, Integrate Microbiology Research Center, South China Agricultural University, Guangzhou, Guangdong 510642, P. R. China. E-mail: yqlin@scau.edu.cn; Fax:+86-20-87518783; Tel: +86-20-85280296

$\uparrow$ Electronic supplementary information (ESI) available. See DOI: 10.1039/c7ra04703g combined contaminants became more difficult to be disposed.

As is known, most organic wastes are degraded by microorganisms in nature and then re-enter into the global carbon cycle system. The synergistic action of microbial consortiums plays an important role in this biodegradation process. Inspired by this view, researchers have constructed different kinds of microbial consortiums for the degradation of lignocellulose, polycyclic aromatic hydrocarbons, pesticides, and phenolic compounds. ${ }^{5}$ In our previous research, we also constructed a microbial consortium named OEM1 that showed a good ability for lignocellulose-rich and chlorophenol-rich organic waste degradation. ${ }^{6}$ The diversity analysis and the degradation characteristics of microbial consortium OEM1 were evaluated. However, issues such as the growth characteristics of this microbial consortium and its stability and potential for degradation need to be addressed.

A number of studies have confirmed that the substrate species, $\mathrm{C} / \mathrm{N}$ ratio, $\mathrm{pH}$, dissolved oxygen, and temperature are important factors for microbial consortium activity. ${ }^{711}$ For example, a composite microbial system MC1 had better degradation efficiency on cellulosic materials (filter paper and absorbent cotton) than on lignocellulosic materials (wheat straw and wood residue). Moreover, previous studies have also explained that $\mathrm{pH}$, temperature, structure of the molecule, and substrate species influence the degradation efficiency for chlorophenols. ${ }^{11}$ Another research confirmed that the dechlorination rates of 2,4,6-TCP and PCP were increased in the presence of dichlorophenols (2,4-DCP and 3,4-dichlorophenol) under anaerobic conditions. ${ }^{12}$ Obviously, an appropriate system 
could stimulate the degradation of lignocellulose and chlorophenols. The degraded substrates could also be utilized for biogas generation via anaerobic digestion.

To develop the degradation potential for lignocellulosic biomass and improve environmental adaptability and operation convenience during the degradation process, the performance of microbial consortium OEM1 under different organic loads, initial $\mathrm{pH}$ values of the culture medium, and cultivating ways were investigated in this study. The rice straw degradation efficiency, lignocellulosic component alteration, chlorophenol removal, $\mathrm{pH}$ and $\mathrm{OD}$ variations, and substrate structure changes have been discussed under various conditions. This study can provide basic scientific data about OEM1 for future commercial application.

\section{Materials and methods}

\section{Materials}

As a microbial consortium, OEM1, with efficient and stable ability for lignocellulose and chlorophenol degradation, was obtained as a lignocellulosic biomass and pulp and paper sludge pretreatment agent in our previous study. ${ }^{6}$ The lignocellulosic material rice straw was obtained after rice harvest from agricultural plots of experimental fields at South China Agricultural University, Guangdong, China. Rice straw was soaked in a $1 \%(\mathrm{w} / \mathrm{v})$ sodium hydroxide solution for 24 hours at room temperature under static condition. After this, the rice straw was washed with distilled water until a neutral $\mathrm{pH}$ was achieved, dried in an oven to a constant weight, then cut into approximately $0.5 \mathrm{~cm}$ length, and stored in a desiccator for further use. O-Chlorophenol (MCP), 2,4-dichlorophenol (2,4-DCP), and 2,4,6-trichlorophenol (2,4,6-TCP), as the three representatives of chlorophenols derived from the pulp and paper sludge, were used in this experiment. The mixed chlorophenol stock solution with ethanol as a solvent and $5.0 \mathrm{~g} \mathrm{MCP}$, 2,4-DCP, and 2,4,6-TCP were added to $100 \mathrm{~mL}$ ethanol. Then, it was stored at $4{ }^{\circ} \mathrm{C}$ in a brown bottle.

\section{Cultivation with the microbial consortium OEM1}

Rice straw and chlorophenols $(50 \mu \mathrm{L}$ mixed chlorophenol stock solution), as the target degradation substrates, were added to the medium. The total concentration of chlorophenols for the degradation experiment was $75 \mathrm{mg} \mathrm{\textrm {L } ^ { - 1 }}$. The enrichment medium contained the following elements per liter of distilled water: $2.5 \mathrm{~g}$ peptone, $1.5 \mathrm{~g} \mathrm{Na} \mathrm{NPO}_{4}, 1.5 \mathrm{~g} \mathrm{KH}_{2} \mathrm{PO}_{4}, 0.8 \mathrm{~g}$ $\mathrm{MgCl}_{2} \cdot 6 \mathrm{H}_{2} \mathrm{O}, 0.8 \mathrm{~g} \mathrm{CaCl} \cdot 2 \mathrm{H}_{2} \mathrm{O}, 0.8 \mathrm{~g}$ yeast extract, and $1 \mathrm{~mL}$ trace element solution. The trace element solution comprised the following compounds ( $\left.\mathrm{g} \mathrm{L}^{-1}\right): \mathrm{ZnCl}_{2}, 0.10 ; \mathrm{MnCl}_{2} \cdot 4 \mathrm{H}_{2} \mathrm{O}, 0.1$; $\mathrm{CaCl}_{2}, 0.20 ; \mathrm{CoCl}_{2} \cdot 6 \mathrm{H}_{2} \mathrm{O}, 0.17 ; \mathrm{H}_{3} \mathrm{BO}_{4}, 0.019 ; \mathrm{Na}_{2} \mathrm{MoO}_{4} \cdot 2 \mathrm{H}_{2} \mathrm{O}$, $0.020 ; \mathrm{NiCl}_{2} \cdot 6 \mathrm{H}_{2} \mathrm{O}, 0.05$; and $\mathrm{CuSO}_{4} \cdot 5 \mathrm{H}_{2} \mathrm{O}, 0.10$. Finally, the $\mathrm{pH}$ was adjusted to neutral. OEM1 from frozen stock was inoculated into $100 \mathrm{~mL}$ of sterile medium for further use. Then, $90 \mathrm{~mL}$ medium containing rice straw and chlorophenol stock solution $(50 \mu \mathrm{L})$ was inoculated with $10 \%(\mathrm{v} / \mathrm{v})$ of the activated OEM1 culture. After this, it was incubated in a $250 \mathrm{~mL}$ flask under dynamic condition (120 rpm) at $28{ }^{\circ} \mathrm{C}$ for 15 days.

\section{Degradation efficiency under different culture conditions}

Different rice straw loads. To investigate the effect of organic load on degradation, $0.5 \mathrm{~g}, 1.0 \mathrm{~g}, 2.0 \mathrm{~g}$, and $4.0 \mathrm{~g}$ rice straw were loaded into a $100 \mathrm{~mL}$ culture system, which made the organic substrate load up to $0.5 \%, 1.0 \%, 2.0 \%$, and $4.0 \%$, respectively, based on total solids (TS). The activated OEM1 (10\%, v/v) was inoculated into the medium. The samples were continuously cultured under shaking condition for 15 days, and the media without inoculation were taken as a control. Herein, twelve replications were carried out for each sample. Optical density (OD) and $\mathrm{pH}$ of the fermentation broth were determined every day. Weight loss of the sample was determined on day 1, 3, 6, 9, 12 , and 15. The degradation of chlorophenols and the lignocellulosic component alteration were analyzed on day 9 and 15 , respectively. Physical structure changes of rice straw were observed via scanning electron microscopy (SEM) after 12 days of treatment.

Different initial $\mathbf{p H}$ values. According to the organic load experiments, an optimal organic load for lignocellulose degradation was selected. The medium $(90 \mathrm{~mL})$ containing $2.0 \mathrm{~g}$ rice straw was adjusted to the initial $\mathrm{pH}$ of values of $5,6,7$, and 8 using dilute $\mathrm{HCl}$ and $\mathrm{NaOH}$ solution before sterilization. The activation OEM1 $(10 \%$, v/v) was inoculated into the medium. The samples were continuously incubated for 15 days under shaking condition. Herein, twelve replications were conducted for each sample. The $\mathrm{pH}$ and $\mathrm{OD}$ of the fermentation broth, weight loss of the rice straw, chlorophenol degradation efficiency, and lignocellulosic component alteration analysis were referred to the OEM1 degradation experiment under different rice straw loads.

Different cultivating ways. To investigate the distinctions in different cultivating modes, two cultivating methods (shaking or stilling) were carried out in this experiment. The organic load in this experiment was $2 \%$. Herein, ten percent of activation OEM1 (v/v) was inoculated into the $90 \mathrm{~mL}$ sterilized medium. The samples were incubated under the shaking $(120 \mathrm{rpm})$ or stilling condition at $28{ }^{\circ} \mathrm{C}$ for 15 days. Each sample was replicated twelve times. The $\mathrm{pH}$ and $\mathrm{OD}$ of fermentation broth, weight loss of the rice straw, chlorophenol degradation efficiency, and lignocellulosic component alteration analysis were referred to the part of different rice straw loads.

\section{Measurement of $\mathrm{pH}, \mathrm{OD}$, and SEM}

About $2 \mathrm{~mL}$ fermentation broth was used for $\mathrm{pH}$ and OD analyses. The methods for $\mathrm{pH}$ and $\mathrm{OD}$ determination were as per the previous literature. ${ }^{9}$ Changes in the physical structure of rice straw were observed via SEM. Dried samples were coated with gold using a sputter coater. Then, they were evaluated using a scanning electron microscope (Philips XL-30) at a voltage of $20 \mathrm{kV}$.

\section{Determination of the weight loss of rice straw and lignocellulosic components}

The weight loss of rice straw was determined via a centrifugalwarm water washing method. The non-lignocellulosic materials were removed by washing three times. Then, the residual 
substrates were oven-dried to a constant weight at $105{ }^{\circ} \mathrm{C}$. The lignocellulosic contents (cellulose, hemicellulose, and lignin) of the samples were determined according to the modified van Soest method. ${ }^{\mathbf{1 3}}$ The details of the modified van Soest method are described in the ESI. $\uparrow$ The degradation rate of each lignocellulosic component was calculated via the following equation: $X_{(k)}=\left(M_{\mathrm{O}(k)}-M_{\mathrm{OEM} 1(k)}\right) / M_{0(k)} . X_{(k)}$ represents the degradation rate of the lignocellulosic component ( $k$ varies with cellulose, hemicellulose, and lignin). $M_{0(k)}$ denotes the mass of the component before degradation. $\operatorname{MOEM} 1(k)$ is the mass of the component after 15 days of degradation by the microbial consortium OEM1.

\section{Determination of the chlorophenol degradation}

Isometric ethanol was added to the medium, and then, it was shaken at $180 \mathrm{rpm}$ ( 1 hour) for the adsorption of chlorophenols by rice straw. ${ }^{\mathbf{1 4}}$ The supernatant liquid was obtained and filtered via a 0.45 micron filter. The degradation of MCP, 2,4-DCP, and 2,4,6-TCP was detected via a high performance liquid chromatograph (HPLC) system with an ODS column. ${ }^{6,14}$ The eluents were methanol and $1 \%$ acetic acid $(78 / 22, \mathrm{v} / \mathrm{v})$ at a flow rate of $1.0 \mathrm{~mL} \mathrm{~min}^{-1}$. The column temperature was $25{ }^{\circ} \mathrm{C}$. The photodiode array detector was used for analysis at $280 \mathrm{~nm}$. The injection volume of the sample was $10 \mu \mathrm{L}$. The degradation rate of chlorophenols was calculated using the following equation: $Y_{(k)}=\left(C_{\mathrm{Ctrl}(k)}-C_{\mathrm{OEM} 1(k)}\right) / C_{\mathrm{Ctrl}(k)}$, where $Y_{(k)}$ represents the degradation rate of chlorophenol ( $k$ varies with MCP, 2,4-DCP, and 2,4,6-TCP), $C_{\mathrm{Ctrl}(k)}$ refers to the concentration of chlorophenol of the control sample after 7 days of culture, and COEM1 $(k)$ denotes the concentration of chlorophenol after 7 days of degradation by microbial consortium OEM1.

\section{Statistical analysis}

Statistical significance was examined via analysis of variance (ANOVA) using SPSS (version 16.0 for Windows.). The probability level at $p<0.05$ was considered significant.

\section{Results and discussion}

\section{The microbial consortium OEM1 for lignocellulose and chlorophenol degradation}

The microbial consortium OEM1 was obtained through successive transfer cultivation for about 50 generations in our previous study. ${ }^{6}$ Preliminary experiments indicated that OEM1 had a good potential for lignocellulose and chlorophenol degradation. OEM1 could degrade more than 50\% rice straw within 15 days of treatment when $0.5 \%(\mathrm{wt} / \mathrm{v})$ rice straw was loaded into the medium. The lignocellulosic components including cellulose, hemicellulose, and lignin were considerably decomposed, and the three-dimensional recalcitrant structure of rice straw was destroyed. The rice straw was significantly decomposed into filament and disintegrated (Fig. 1). Moreover, in the later screening stage, MCP, 2,4-DCP, and 2,4,6-TCP were removed after 7 days of treatment by OEM1. ${ }^{6}$ These degradation features of OEM1 depicted the bright prospect of OEM1 in the disposal of agricultural and forestry wastes,
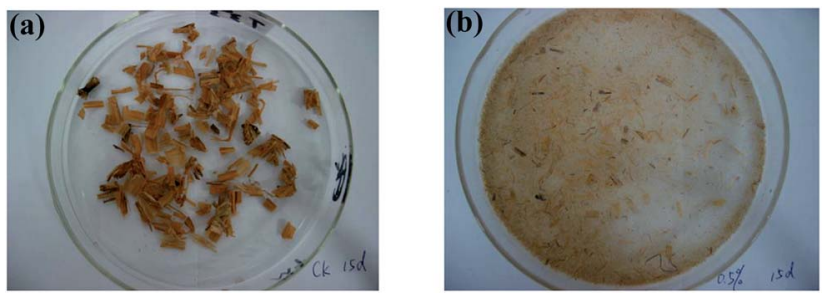

Fig. 1 Rice straw appearance alteration after OEM1 treatment. (a) Control sample and (b) the sample treated with OEM1 for 15 days.

and it could be applied in lignocellulosic biomass composting to accelerate lignocellulose decomposition and shorten the composting cycle. Moreover, the microbial consortium OEM1 could be utilized as a pretreatment agent for lignocellulosic biomass. In our previous research, we have reported that biogas production derived from rice straw and pulp and paper sludge co-anaerobic digestion was significantly increased via microbial consortium OEM1 pretreatment for 9 days. ${ }^{15}$ To further evaluate the potential of OEM1 for practical application, its organic load, acid/alkali stability, and cultivation ways have been proposed hereinafter.

\section{Effects of culture conditions on rice straw degradation}

As shown in Fig. 2(a), the rice straw degradation efficiency increased when the organic load increased from $0.5 \%$ to $2.0 \%$. Within 15 days of treatment, the dry mass loss of rice straw was $55.91 \%, 63.79 \%$, and $76.50 \%$, corresponding to the samples of organic load of $0.5 \%, 1.0 \%$, and $2.0 \%$, respectively. This could be attributed to the nutritional structure improvement $(\mathrm{C} / \mathrm{N}$ ratio). However, the rice straw degradation rate significantly decreased (only to $42.92 \%$ ) when the organic load was increased to $4.0 \%$. It was probably because high concentration of organic substrate ( $\mathrm{C} / \mathrm{N}$ ratio) inhibited the growth of the microbial consortium OEM1 and the rice straw degradation function. The results illustrated that highest degradation rate of

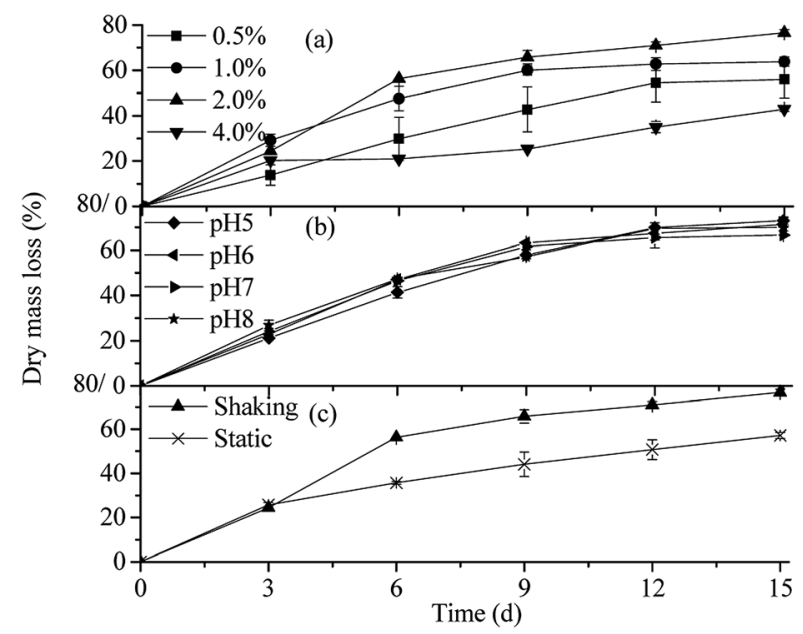

Fig. 2 Dry matter loss of rice straw in different culture situations ((a) organic loads; (b) initial $\mathrm{pH}$ values; and (c) cultivating ways). 
lignocellulose was obtained when the organic load was $2.0 \%$. Compared to the degradation rate of rice straw after 15 days of treatment, more than $50 \%$ rice straw was degraded in the period of exponential phase of the microbial consortium OEM1 (0-6 d). In this period, microbial consortium rapidly grew (shown in Fig. 5) and exhibited high lignocellulosic degradation activity. The characteristics of rice straw degradation were similar to those of the rice straw decomposed by the bacteria community NSC-7, which could degrade cellulose and lindane (decomposed more than $50 \%$ in the initial 6 days). ${ }^{9}$ Surprisingly, rapid degradation of rice straw was noted in the first three days when the organic load increased to $4.0 \%$. In the first three days, the microbial consortium OEM1 rapidly grew, and the OD value rapidly increased (Fig. 5(a)). The microbial consortium OEM1 rapidly grew at the beginning and utilized the organic substrates; this probably led to the high degradation rate of rice straw in the first three days.

At different initial $\mathrm{pH}$ values, the degradation rates of rice straw treated by OEM1 for 15 days were higher than $65 \%$. The rice straw degradation efficiency was not influenced by acidification of the medium. The rice straw in the reactor with $\mathrm{pH} 5$ was degraded by $72.99 \%$ within 15 days. As shown in Fig. 2(b), OEM1 exhibited high degradation efficiency for rice straw no matter whether the condition was acidic or alkaline. It was suggested that OEM1 had a good lignocellulose degradation and $\mathrm{pH}$ buffer capacity, which were conducive to its practical application in a complicated environment.

Via shaking cultivation, $76.50 \%$ rice straw was degraded in 15 days, whereas $57.09 \%$ rice straw was degraded via static cultivation. It may correspond to the rich dissolved oxygen in the shaking cultivation process because OEM1 can rapidly grow and proliferate in the oxygen-rich culture environment and its capability for lignocellulose degradation enhances. ${ }^{10}$ However, obvious advantages of using static cultivation are as follows: (i) simple operation and (ii) less equipment. In practical applications, it can be combined with actual requirements to select a suitable cultivation way.

\section{Effects of the culture conditions on the lignocellulosic component degradation}

The degradation of the lignocellulosic components (cellulose, hemicellulose, and lignin) is plotted in Fig. 3. After 15 days of degradation, highest efficiency for lignocellulosic component degradation was obtained when $2.0 \%$ rice straw was loaded into the culture medium. Cellulose, hemicellulose, and lignin were degraded by $97.40 \%, 76.44 \%$, and $69.79 \%$, respectively. The result was consistent with the highest degradation efficiency for rice straw obtained from the sample with $2 \%$ organic load. Cellulose, hemicellulose, and lignin in the reactors with $0.5 \%$ and $1.0 \%$ organic load were degraded by about $90 \%, 65 \%$, and $60 \%$, respectively. However, the lignocellulosic component degradation with $4 \%$ organic load was lowest, in line with the lowest dry matter loss observed in these reactors. Compared to those of the $2 \%$ organic load sample, the degradation rates of cellulose, hemicellulose, and

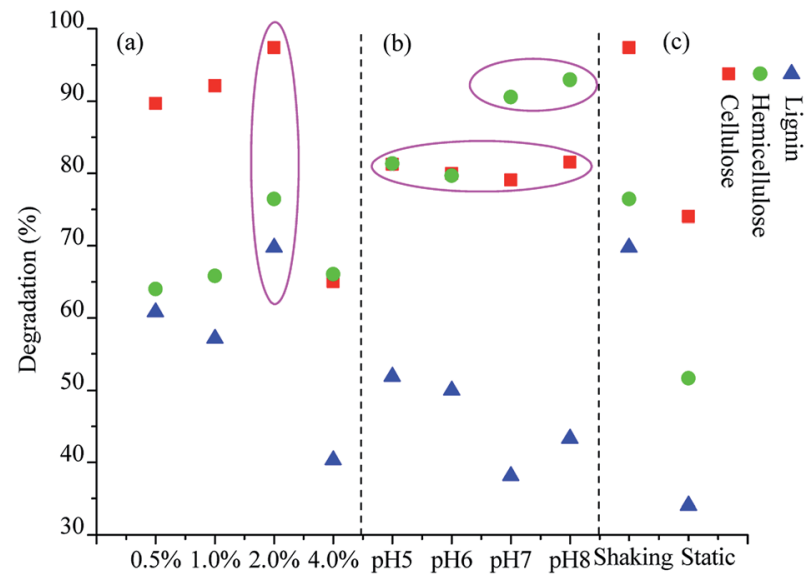

Fig. 3 Lignocellulosic component degradation under different culture conditions ((a) organic loads; (b) initial pH values; and (c) cultivating ways).

lignin were decreased by $32 \%, 10.36 \%$, and $29.43 \%$, respectively.

The degradation rate of cellulose with different initial $\mathrm{pH}$ values were about $80 \%$. The degradation rates of hemicellulose at the initial pH 7 and pH 8 reached $90.6 \%$ and 93\%, respectively, which were $11.36 \%$ and $13.22 \%$, respectively, higher than those obtained at the initial pH 5 and pH 6. On the contrary, the degradation rates of lignin at the initial pH 5 (51.92\%) and pH 6 $(49.98 \%)$ were $13.78 \%$ and $6.65 \%$, respectively, higher than those obtained at the initial $\mathrm{pH} 7$ and $\mathrm{pH}$ 8. The results indicated that the weak alkaline medium was favorable for hemicellulose degradation, and the weak acidic medium was beneficial for lignin degradation. These findings may be due to the alteration of consortium community structure in different culture situations, and our previous research has confirmed that $\mathrm{pH}$ plays an important role in the dynamic evolution of OEM1. ${ }^{6}$ Our further studies will be focused on the dynamic changes on the consortium community structure of the microbial consortium OEM1 in different culture situations via real-time quantitative PCR.

Furthermore, OEM1 exhibited a better ability for lignin degradation as compared to the composite microbial system MC1 reported by Wang et al. ${ }^{16}$ Through shaking cultivation, OEM1 could degrade $70 \%$ lignin of rice straw within 15 days, whereas MC1 could only degrade 51\% lignin under the similar culture condition. However, the lignin degradation by static cultivation was only $34 \%$, much lower than that obtained by shaking cultivation.

Considering all the culture conditions (rice straw organic loads, initial $\mathrm{pH}$ values, and cultivating ways), the average degradation rate for cellulose and hemicellulose was $32.22 \%$ and $22.97 \%$, respectively, higher than that of lignin. This suggested that the utilization of the decomposed cellulose and hemicellulose was easier than that of lignin. However, the degradation of lignin was over $50 \%$. It was clear that more than half of the compact lignocellulosic structure connected by cellulose, hemicellulose, and lignin had been broken down. It indicated that OEM1 had a significant influence on the 
breakage of the biomass recalcitrance and biomass utilization.

\section{Effects of culture conditions on the chlorophenol degradation}

The degradation of chlorophenols by OEM1 after 9 days of cultivation under different culture conditions is depicted in Fig. 4. OEM1 exhibited high efficiency for chlorophenol degradation when the organic loads were $0.5 \%$ and $2 \%$. MCP, 2,4-DCP, and 2,4,6-TCP were almost completely degraded. The degradation rate of 2,4,6-TCP with $0.5 \%$ organic load was higher than that with $2 \%$ organic load. This could be explained by two reasons: (i) OEM1 was screened with $0.5 \%$ organic load and (ii) the lignocellulose degradation strains in OEM1 became dominant when the rice straw organic load increased. In addition, the sample with $1.0 \%$ organic load had good degradation ability for chlorophenols. MCP and 2,4-DCP were totally degraded after 9 days, and 2,4,6-TCP was degraded by $45.81 \%$. However, the degradations of MCP, 2,4-DCP, and 2,4,6-TCP were $21.53 \%$, $87.57 \%$, and $77.87 \%$, respectively, when $4 \%$ rice straw was loaded into the medium.

As shown in Fig. 4(b), OEM1 exhibited obvious distinctions for chlorophenol degradation at different initial $\mathrm{pH}$ values. The degradation rate of chlorophenols at the initial pH 7 (97.04\%) and $\mathrm{pH} 8(89.37 \%)$ were $35.24 \%$ and $22.95 \%$, respectively, higher than those obtained at the initial $\mathrm{pH} 5$ and $\mathrm{pH} 6$. Moreover, the initial $\mathrm{pH} 7$ sample showed best performance for chlorophenol degradation. The results illustrated that higher chlorophenol degradation efficiency was noted under neutral and alkalescent conditions as compared to that obtained under weakly acidic conditions. This was because the dechlorination reaction of chlorophenols as a result of nucleophilic substitution was easier under alkaline conditions. ${ }^{17}$

The chlorophenol degradation by OEM1 via shaking cultivation was superior to that via static cultivation. MCP and 2,4-DCP were not detected after 9 days of degradation under shaking cultivation. The degradation rate of 2,4,6-TCP reached 91.93\%. With static cultivation for 9 days, 2,4-DCP was not detected. The degradation rates of MCP and 2,4,6-TCP were $13.99 \%$ and $61.88 \%$, respectively. The total amount of

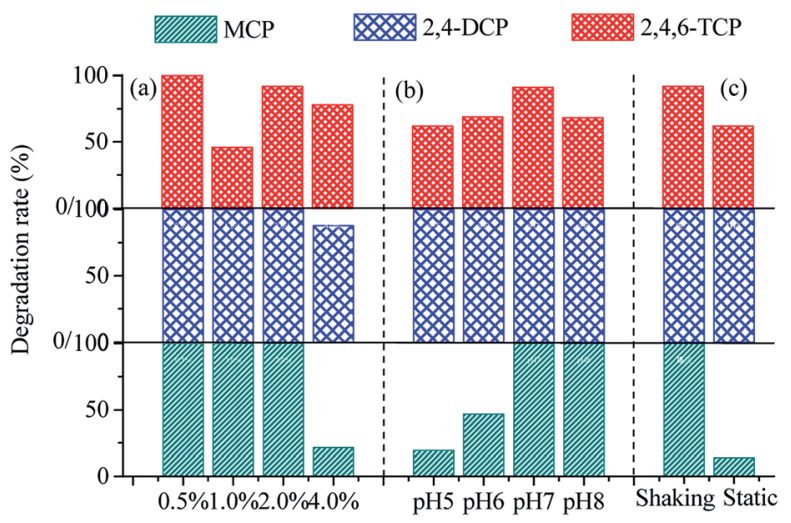

Fig. 4 Chlorophenol degradation in different culture situations ((a) organic loads; (b) initial pH values; and (c) cultivating ways).

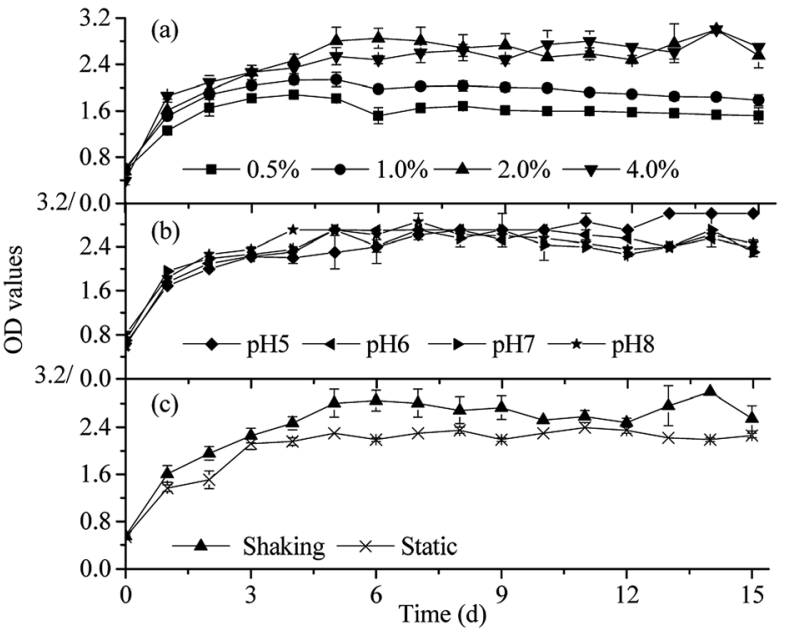

Fig. 5 Curves of the OD values in different culture situations ((a) organic loads; (b) initial pH values; and (c) cultivating ways).

chlorophenols degraded was approximatively $60 \%$. The results highly agreed with the growth characteristics of OEM1 (Fig. 5) and the degradation efficiency of rice straw (Fig. 2 and 3).

On comparing the degradation efficiency of MCP, 2,4-DCP, and 2,4,6-TCP under different culture conditions, it was observed that the degradation efficiency of 2,4-DCP was superior to those of MCP and 2,4,6-TCP. It indicated that OEM1 had a better selectivity for 2,4-DCP degradation. Generally, the higher the degree of chlorine substitution in phenol, the more the toxicity of chlorophenol. The toxicities of 2,4,6-TCP and 2,4-DCP are higher than that of MCP; therefore, their chemical structure is more stable. However, a previous research indicated that co-metabolism effect could affect the biodegradation process of chlorophenols. ${ }^{18}$ Quan et al. ${ }^{19}$ suggested that the removal of 2,4-DCP was strengthened in an activated sludge system after the addition of 4-monochlorophenol for bioaugmentation in the system. Herein, high decomposition efficiency for 2,4-DCP may be due to 2,4-DCP and MCP exhibiting co-metabolism effects in early degradation period.

\section{Variations of the OD values in the degradation process}

Fig. 5 shows the variations of microbial biomass of OEM1 based on OD under different culture conditions. In the first 5 days, OEM1 was in the exponential phase, and OD values rapidly increased. It was consistent with the result of $56.29 \%$ rice straw degradation in the initial 6 days (Fig. 2). Then, the growth of OEM1 went into a balanced phase (6-15 d), and the OD values for $0.5 \%, 1.0 \%, 2.0 \%$, and $4.0 \%$ were maintained at around 1.6 , 2.0, 2.7, and 2.7, respectively. As shown in Fig. 5(a), with the increasing organic load from $0.5 \%$ to $2.0 \%$, the OD values simultaneously increased. This was probably due to the increase of available organic carbon with the increasing organic load. However, the OD values did not increase when the organic load was $4.0 \%$; this indicated that microbial proliferation was inhibited. It was most likely owing to the high $\mathrm{C} / \mathrm{N}$ ratio of the cultivation system and organic acid accumulation (showed in 


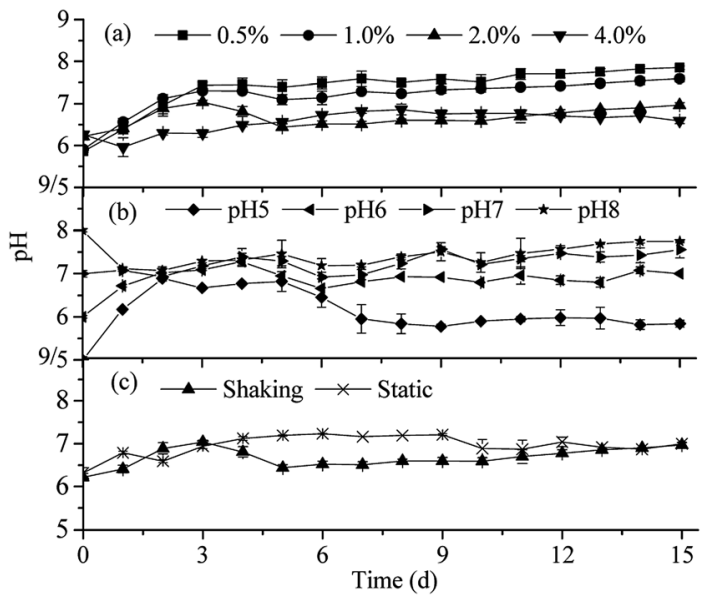

Fig. 6 Curves of $\mathrm{pH}$ values in different culture situations ((a) organic loads; (b) initial $\mathrm{pH}$ values; and (c) cultivating ways).

Fig. 6(a)). The result could guide the trophic structure of substrate optimization and contribute to the growth of microorganisms.

The growth characteristics of OEM1 at different initial $\mathrm{pH}$ values were similar. In the first 3 days, the OD values rapidly increased to about 2.2. Then, OEM1 slowly proliferated, and the OD values maintained at about 2.7 after 6 days of cultivation. The OD values in the shaking cultivation way were $15.21 \%$ higher than those in the static cultivation way (Fig. 5(c)) during the degradation process. It indicated that the proliferation of OEM1 under shaking cultivation way was faster than that under the static cultivation way. The result was consistent with the higher lignocellulose and chlorophenol degradation efficiency with OEM1 cultivated via the shaking cultivation way. The OD values of the shaking and static cultivation ways were maintained at around 2.7 and 2.3 after 5 days of inoculation, respectively.

\section{Variations of $\mathrm{pH}$ values in the degradation process}

The variations of $\mathrm{pH}$ values of the fermentation broth during the degradation process under different culture conditions are shown in Fig. 6 . When $0.5 \%$ and $1 \%$ rice straw were loaded into the medium, the $\mathrm{pH}$ values of the fermentation broth increased from initial 5.90 to around 7.30 within 3 days. A slight decline was noted on day 5 , and then, the $\mathrm{pH}$ value slowly began to increase on day 7; finally, it remained at around 8.00 till the end of the culture. The $\mathrm{pH}$ values in the $2.0 \%$ organic load sample increased to the peak (7.04) in the first 3 days and then rapidly dropped to 6.44 in the following two days. After this, the $\mathrm{pH}$ values slowly increased and finally reached the neutral value on day 13 . With regard to $4.0 \%$ rice straw loaded into the medium, the $\mathrm{pH}$ values dropped from the initial 6.26 down to 5.96 after inoculation within 24 hours. Since then, it slowly went up and remained at about 6.70 on day 6 . For the samples with $0.5 \%$, $1 \%$, and $2 \%$ organic load, the variation trends of $\mathrm{pH}$ values were similar to those of the $\mathrm{pH}$ characteristics of the composite microbial system RSS-4 obtained by Liu. ${ }^{20}$ However, it was opposite to that of the composite microbial systems obtained by Cui. $^{7,17}$ It may be due to the different components of the medium. The medium in this experiment contained lots of $\mathrm{HPO}_{4}{ }^{-2}$ and $\mathrm{H}_{2} \mathrm{PO}_{4}{ }^{-1}$, which caused an acidic environment at the beginning. After the proliferation of OEM1, the peptone and beef extract of the medium rich in nitrogen were utilized by microorganisms. The alkaline secretions were accumulated, and then, the $\mathrm{pH}$ value increased. Thereafter, organic acids and other substances increased as a consequence of the rice straw decomposition; this caused the $\mathrm{pH}$ value to decrease. As the organic load increased, more acidic metabolites were accumulated. Therefore, the $\mathrm{pH}$ values of $2.0 \%$ and $4.0 \%$ organic load samples were lower than those of $0.5 \%$ and $1.0 \%$. However, acid inhibition was not noted in the $2.0 \%$ and $4.0 \%$ organic load samples. The $\mathrm{pH}$ values of $2.0 \%$ and $4.0 \%$ organic load samples were almost unanimous after 5 days of inoculation. It suggested that the overabundant substances of the $4.0 \%$ organic load samples were not fully utilized by OEM1; this was consistent with the characteristics of OEM1 growth and organic substance degradation.

The $\mathrm{pH}$ of the fermentation broth became neutral after two days of inoculation including that of the samples with the initial $\mathrm{pH}$ values ranging from 5.0 to 8.0. It indicated that OEM1 had strong acidic and alkaline buffer capacity. After this, the samples with the initial $\mathrm{pH}$ values of $6.0,7.0$, and 8.0 became stable at around 7.0, except a weak fluctuation on day 6 . However, the $\mathrm{pH}$ value of the sample with the initial $\mathrm{pH}$ value of 5 slowly dropped down to 6.0 on day 7 and finally maintained at around 5.9. The $\mathrm{pH}$ value of this sample was lowest as compared to that of others. This was probably due to highest efficiency of the sample with the initial $\mathrm{pH}$ value of 5 for lignocellulose degradation (Fig. 2 and 3), and this led to more acidic metabolites formation (e.g. acetic acid).

Whether cultivated via shaking or static cultivation way, the $\mathrm{pH}$ changing tendency of OEM1 was similar. The $\mathrm{pH}$ values first increased after OEM1 inoculation and then dropped slightly as a result of lignocellulose decomposition and acidic metabolite accumulation. Then, it gradually increased again and finally remained at about 7.0. As Fig. 6(c) shows, the $\mathrm{pH}$ values of the sample cultivated via shaking lowered down after the 3 days of culture. It might be ascribed to higher degradation efficiency of rice straw, which produced more organic acid products within the reactor.

\section{Physical structure alteration of rice straw}

The physical structure of rice straw after degradation by OEM1 was observed via SEM. The images are presented in the ESI Fig. S1. $\uparrow$ The raw rice straw exhibited a continuous and smooth surface covered with a wax layer. Moreover, the cells, stomata, epidermal appendages, and epidermal trichomes embedded in the siliceous layer were regularly distributed. Rice straw was significantly altered after 12 days of degradation. There were lots of hyphal coils and cells stuck to the surface. The SEM analysis indicated that the rice straw was decomposed most seriously when $2.0 \%$ rice straw was loaded into the medium. The trichomes of the outer surface were almost completely 
degraded, and the epidermal tissues were exposed. The parenchyma was destroyed; this caused the vascular bundle to be separated from the mechanical tissue and the structure of fiber bundle to become loose. With regard to the sample with 1.0\% organic load, a majority of siliceous layer was destroyed, the structure of the fiber bundle became loose, and the dense structure was destroyed. Compared to the $0.5 \%$ and $4 \%$ organic load samples, the $1.0 \%$ organic load sample exhibited better degradation capacity, and the siliceous layer of the surface became thinner and lignocellulose was partly degraded. The SEM results agreed with the tendency of lignocellulose disposed by OEM1 with different organic loads, and the $2.0 \%$ organic load sample was optimal, followed by $1.0 \%, 0.5 \%$, and $4.0 \%$ organic load samples.

\section{Conclusion}

OEM1 showed great ability for lignocellulose and chlorophenol degradation. The optimal organic load of the rice straw was $2.0 \%$, resulting in the dry mass loss of $76.50 \%$ (within 15 days) and the chlorophenol degradation efficiency of over $95 \%$ (within 9 days). Moreover, more than 65\% of rice straw was degraded when the initial $\mathrm{pH}$ value was in the range of 5.0-8.0; this indicated that OEM1 had a good adaptability in the alkaline/acidic system. Compared to the case of shaking cultivation, the degradation of lignocellulose and chlorophenols via static cultivation could still reach $57.1 \%$ and $58.6 \%$, respectively. This study provides a significant disposal method or pretreatment for lignocellulosic biomass (agricultural and forestry wastes or industrial wastes) utilization.

\section{Acknowledgements}

This work was supported by the National Natural Science Foundation of China (No. 51108195) and Science and Technology Project of Guangdong Province under Contract No. 2015A010106012 and 2016A020210085.

\section{Notes and references}

1 T. Mahmood and A. Elliott, Water Res., 2006, 40, 2093-2112.
2 J. M. Reckamp, R. A. Garrido and J. A. Satrio, Biomass Bioenergy, 2014, 71, 235-244.

3 P. Bajpai, Environmentally Benign Approaches for Pulp Bleaching, Elsevier, USA, 2nd edn, 2012.

4 D. Savant, R. Abdul-Rahman and D. Ranade, Bioresour. Technol., 2006, 97, 1092-1104.

5 H. P. Bacosa and C. Inoue, J. Hazard. Mater., 2015, 283, 689697.

6 J. Liang, X. Peng, D. Yin, B. Li and Y. Lin, Bioresour. Technol., 2015, 190, 381-387.

7 Z. Cui, M. Li, Z. Piao, Z. Huang, M. Ishii and Y. Igarashi, Environ. Sci., 2002, 23, 36-39.

8 Z. Cui, Z. Piao, W. Wang, B. Su and H. Yu, J. Agr. Environ. Sci., 2004, 23, 296-299.

9 C. Liu, X. Wang, J. Niu, X. Li, H. Shen and Z. Cui, Microbiol. China, 2008, 35, 725-730.

10 P. Lu, G. Li, L. Chen, G. Wang, X. Wang and Z. Cui, Trans. Chin. Soc. Agric. Eng., 2008, 24, 209-213.

11 A. O. Olaniran and E. O. Igbinosa, Chemosphere, 2011, 83, 1297-1306.

12 B. V. Chang, K. T. Liao and S. Y. Yuan, Environ. Chem., 1995, 49, 33-43.

13 S. Liu, Dissertation, Chinese Academy of Agricultural Sciences, 2011.

14 A. Khodadoust, M. Suidan, C. Acheson and R. Brennerl, Chemosphere, 1999, 38, 2681-2693.

15 Y. Lin, L. Liang, C. Zeng, D. Wang and H. Lin, Renewable Energy, 2017, 108, 108-115.

16 W. Wang, Z. Cui, X. Wang, J. Niu, J. Liu and Y. Igarashi, Environ. Sci., 2005, 26, 156-160.

17 J. Niu, G. Li, Z. Cui, W. Wang and J. Liu, Environ. Sci., 2005, 26, 186-190.

18 G. Baggi, V. Andreoni, S. Bernasconi, L. Cavalca and M. Zangrossi, Ann. Microbiol., 2002, 52, 133-143.

19 X. Quan, Z. Yang, H. Shi, Q. Tang and Y. Qian, Process Biochem., 2005, 40, 3462-3467.

20 J. Liu, L. Li, H. Chen, D. Guan, X. Jiang, J. Li and D. Shen, Microbiol. China, 2010, 37, 1293-1298. 